\title{
Decolourisation of Remazol Brilliant Blue R via a novel Bjerkandera sp. strain
}

\author{
Patrícia R. Moreira a,b, E. Almeida-Vara ${ }^{\text {a }}$, G. Sena-Martins ${ }^{\text {a }}$, I. Polónia ${ }^{\text {a,c }}$, \\ F. Xavier Malcata ${ }^{b}, *$ J. Cardoso Duarte ${ }^{a}$
}

\author{
Keywords: Bjerkandera sp.; Remazol Brilliant Blue R; Dyes \\ a Unidade de Bioengenharia e Bioprocessos, Instituto Nacional de Engenharia e Tecnologia Industrial (INETI), \\ Estrada do Paço do Lumiar, 22, P-1649-038 Lisboa, Portugal \\ ${ }^{\mathrm{b}}$ Escola Superior de Biotecnologia, Universidade Católica Portuguesa, R. Dr. António Bernardino de Almeida, \\ P-4200-072 Porto, Portugal \\ ${ }^{\mathrm{c}}$ Escola Superior Agrária de Santarém, Quinta do Galinheiro, S. Pedro, P-2000-014 Santarém, Portugal
}

\begin{abstract}
A novel strain of Bjerkandera sp. (B33/3), with particularly high decolourisation activities upon Poly R-478 and Remazol Brilliant Blue R (RBBR) dyes, was isolated. The role of the ligninolytic extracellular enzymes produced by this strain on decolourisation of RBBR was studied in some depth. The basis of decolourisation is an enzyme-mediated process, in which the main enzyme responsible is a recently described peroxidase with capacity for oxidation of manganese, as well as veratryl alcohol and 2,6-dimethoxyphenol in a manganese-independent reaction. (C) 2001 Elsevier Science B.V. All rights reserved.
\end{abstract}

\section{Introduction, results and discussion}

Decolourisation of specific dyes, e.g. Remazol Brilliant Blue R (RBBR) and Poly R, has been used since the eighties as a rapid screening method for detection of ligninolytic ability in fungal strains. Dye decolourisation has been ascribed by several authors to peroxidases produced by said fungi, namely lignin peroxidases, man-

\footnotetext{
* Corresponding author. Tel.: + 351-22-558-0004; fax: + 351-22-509-0351.

E-mail address: xmalcata@esb.ucp.pt (F. Xavier Malcata).
}

ganese peroxidases and laccases (Pasti and Crawford, 1991; Ollikka et al., 1993, 1998; Spadaro and Renganathan, 1994; Knapp et al., 1995; Vyas and Molitoris, 1995; Kim et al., 1996; Shin et al., 1997; Azmi et al., 1998; Heinfling et al., 1998a,b,c; Swamy and Ramsay, 1999).

Recently, a novel class of ligninolytic peroxidases, with high affinity for manganese and dyes, has been described; these enzymes can also oxidise 2,6-dimethoxyphenol (DMP) and veratryl alcohol in a manganese-independent reaction. Those versatile enzymes thus possess a high biotechnological interest because of their broad substrate 
specificity, as well as lack of requirement for mediators to efficiently oxidise and decolourise several dyes; however, they have only been described to exist in Pleurotus sp. and Bjerkandera sp. (Heinfling et al., 1998a,b,c; Mester and Field, 1998; Camarero et al., 1999; Ruiz-Duenas et al., 1999; Palma et al., 2000).

In this work, the ability to decolourise RBBR by a new Bjerkandera sp. strain, obtained from ligninocellulosic rot material, was studied; the focus was on the efficiency of decolourisation, but encompassed also the agents responsible for it in attempts to shed further light onto the physiological performance of Bjerkandera sp. via specific characterization of the recently described $\mathrm{LiP}_{-}$ $\mathrm{MnP}$ hybrid peroxidase.

The new isolate of Bjerkandera sp., strain B33/ 3, was selected from within the collection of strains of Aspergillus niger, Ceriporiopsis subvermispora, Paecylomyces variotti, Penicillium frequentans, Penicillium spinolosum, Phanerochaete chrysosporium, Pleurotus ostreatus, Pycnoporus cinnabarinus and another 109 fungal isolates not yet identified taxonomically, via a screening procedure for RBBR- and Poly-R 478-decolourisation ability.

The aforementioned screening procedure was made in both solid medium, viz. CDAYE medium composed of $45.4 \mathrm{~g}^{-1}$ of Czapex Dox modified Agar (Oxoid) and $5 \mathrm{~g}^{-1}$ of yeast extract (Difco), and liquid medium, viz. CDBYE medium composed of $33.4 \mathrm{~g}^{-1}$ of Czapex Dox modified liquid medium (Oxoid) and $5 \mathrm{~g}^{-1}$ of yeast extract (Difco), both supplemented with $0.05 \%(\mathrm{~m} / \mathrm{v})$ RBBR (Sigma) or $0.02 \% \quad(\mathrm{~m} / \mathrm{v})$ Poly-R 478 (Sigma). The novel strain of interest was selected for its ability to rapidly and efficiently decolourise both dyes in the experimental conditions used; the remaining strains decolourised only one of the dyes, or their rate of decolourisation of both was excessively slow.

The novel fungal strain was originally isolated from rotting ligninocellulosic material, and was duly identified by the Centraalbureau voor Schimmelcultures (Institute of the Royal Netherlands Academy of Arts and Sciences, Baarn, The Netherlands) as belonging to the Bjerkandera genus; it was tentatively named Bjerkandera sp. strain $\mathrm{B} 33 / 3$.
Cultivations in agitated liquid medium (CDBYE) with Bjerkandera sp. strain B33/3 in the presence of RBBR indicated that decolourisation was not noticeable until $5 \mathrm{~d}$ and achieved a maximum of $96 \%$ decolourisation by $11 \mathrm{~d}$. Swamy and Ramsay (1999) reported that Bjerkandera sp. strain BOS55, in agitated liquid culture, achieved a maximum of $65 \%$ decolourisation for the same dye but only by $20 \mathrm{~d}$ and was thus considerably less efficient than our strain.

In a parallel run, cultures of strain B33/3 were tested with and without RBBR in the culture medium. Under the experimental conditions used, that fungal strain exhibited ligninolytic activities typically associated with lignin peroxidase, manganese-dependent peroxidase and manganeseindependent peroxidase, in the presence and in the absence of RBBR; said activities reached their maxima between 5 and $7 \mathrm{~d}$. Table 1 tabulates the enzymatic activities determined in the crude extract for the culture grown for $5 \mathrm{~d}$ in medium without RBBR.

Our results do in general agree with those reported by several authors that have worked with Bjerkandera sp. Unlike the well-studied model organism $P$. chrysosporium, our strain is $N$-unregulated, and produces significant amounts of ligninolytic enzymes as a secondary metabolic event triggered by the discontinuation of growth due to carbon limitation (Kaal et al., 1993; Kotterman et al., 1996; Mester et al., 1996; Mester and Field, 1997).

The detection of the manganese-independent peroxidase activity is also in agreement with the realization that $\mathrm{MnP}$ isoenzymes of the Pleurotus sp. and Bjerkandera sp. differ from those isolated from $P$. chrysosporium because they are able to oxidize DMP and veratryl alcohol in a manganese-independent reaction (Heinfling et al., 1998a,b,c; Mester and Field, 1998; Camarero et al., 1999; Ruiz-Duenas et al., 1999; Palma et al., 2000).

When RBBR was present in the culture medium, the maximum activity of the ligninolytic enzyme MnP (43 IU $1^{-1}$ ) was higher than that reported by Heinfling et al. (1998c), viz. 23 IU $1^{-1}$, for a Bjerkandera adusta strain grown in glucose-ammonium medium added with the azo 
Table 1

Enzymatic activities of the crude extract of Bjerkandera sp. strain B33/3 before and after desalting

\begin{tabular}{|c|c|c|c|c|}
\hline \multirow[t]{2}{*}{ Type of enzymatic activity } & \multicolumn{2}{|c|}{ Activity \pm S.D. $\left(\mathrm{IU}^{-1}\right)$} & \multicolumn{2}{|c|}{ Specific activity \pm S.D. $\left(\text { IU } \mathrm{mg}^{-1}\right)^{\mathrm{d}}$} \\
\hline & Crude extract & Desalted extract & Crude extract & Desalted extract \\
\hline $\mathrm{LiP}^{\mathrm{a}}$ & $220 \pm 1$ & $94 \pm 11$ & $7.6 \pm 0.1$ & $2.5 \pm 0.3$ \\
\hline $\mathrm{MnP}^{\mathrm{b}}$ & $36 \pm 4$ & $27 \pm 0$ & $1.2 \pm 0.1$ & $0.7 \pm 0.0$ \\
\hline $\mathrm{MiP}^{\mathrm{c}}$ & $62 \pm 8$ & $44 \pm 2$ & $2.1 \pm 0.2$ & $1.2 \pm 0.1$ \\
\hline
\end{tabular}

S.D., standard deviation.

${ }^{a}$ The activity of lignin-peroxidase (LiP, EC 1.11.1.14) was determined with veratryl alcohol (Aldrich) as substrate following the method by Linko and Haapala (1993). One unit of enzyme activity (IU) is defined as the amount of enzyme that transforms $1 \mu$ mole of substrate per minute.

${ }^{b}$ The activity of manganese-peroxidase (MnP, EC 1.11.1.13) was determined with MBTH (Sigma) and DMAB (Sigma) as substrates following the method by Castillo et al. (1994). One unit of enzyme activity (IU) is defined as the amount of enzyme that transforms $1 \mu$ mole of substrate per minute.

${ }^{\mathrm{c}}$ The manganese-independent peroxidase activity (MiP) was determined with DMP (Sigma) as substrate following the method by Mester and Field (1997). One unit of enzyme activity (IU) is defined as the amount of enzyme that transforms $1 \mu$ mole of substrate per minute.

d Protein was measured with the Bio-Rad Protein Assay (Bio-Rad Laboratories), according to the manufacturer's instructions, using bovine serum albumin (Fluka) as standard.

dye reactive violet 5 . Furthermore, and in contrast with Heinfling et al. (1998c), it was possible to detect the activity of the ligninolytic enzyme LiP, which reached a maximum of $111 \mathrm{IU} 1^{-1}$. One unit of enzyme activity (IU) was defined as the amount of enzyme that oxidises $1 \mu$ mole of RBBR per min.

For the RBBR decolourisation activity studies, the crude extract of strain B33/3 was previously desalted by passing through Sephadex G-25 PD10 (Amersham Pharmacia Biotech) so as to avoid the presence of $\mathrm{Mn}(\mathrm{II})$, veratryl alcohol or small molecular weight compounds that might interfere with the assay. Table 1 includes the enzymatic activities determined after desalting.

The influence of RBBR concentration upon the decolourising activity of strain B33/3 is illustrated in Fig. 1. The RBBR decolourisation rate increased (as expected) with increases in substrate concentration, from $5 \mu \mathrm{M}\left(26.44 \mathrm{IU}^{-1}\right)$ up to 25 $\mu \mathrm{M}\left(43.51 \mathrm{IU}^{-1}\right)$. Further increases in RBBR concentrations led to inhibition: $105 \mu \mathrm{M}$ RBBR actually led to an activity that was $32 \%$ below the maximum obtained. Vyas and Molitoris (1995), using crude extract from a $P$. ostreatus strain, claimed that the RBBR decolourising activity follows Michaelis-Menten kinetics: its rate increased until a plateau of $100.5 \mu \mathrm{M}$ RBBR was reached; this concentration is, however, higher than the one that produced the plateau with our strain (i.e.

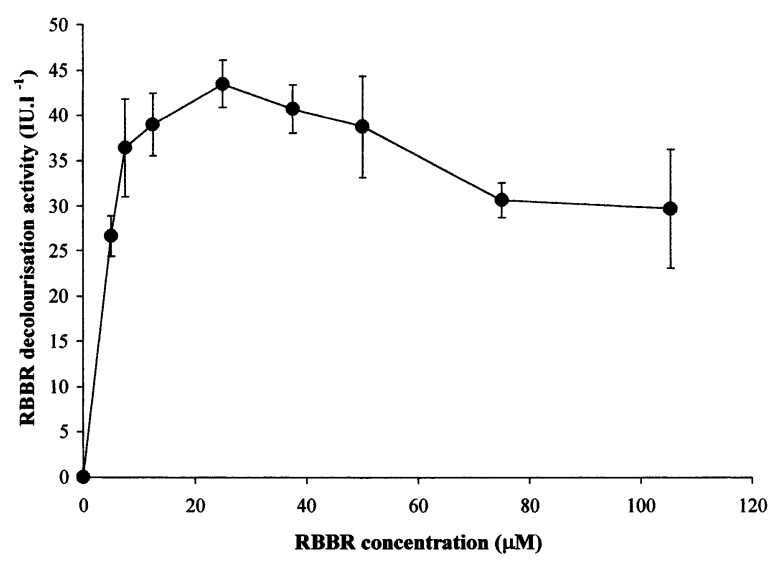

Fig. 1. Influence of concentration of substrate (mean and standard deviation) upon RBBR decolourisation activity by the crude extract of Bjerkandera sp. strain B33/3. The RBBR decolourising activity was assayed spectrophotometrically by measuring the decrease in absorbance at $595 \mathrm{~nm}$ and $30^{\circ} \mathrm{C}$. The enzymatic standard reaction mixture consisted of 0.05 $\mathrm{mM}$ RBBR and $70 \mathrm{mM}$ sodium tartrate buffer $(\mathrm{pH} 3.5)$. The reaction was initiated via addition of $0.1 \mathrm{mM} \mathrm{H}_{2} \mathrm{O}_{2}$. The RBBR decolourising activity was calculated from the time slope of the first linear stage of reaction, using as molar extinction coefficient $\varepsilon_{595}=8266 \mathrm{M}^{-1} \mathrm{~cm}^{-1}$. 
$25 \mu \mathrm{M}$ ). Using purified peroxidase isoenzymes from Pleurotus sp. strains and Bjerkandera sp. strains, Shin et al. (1997) and Heinfling et al. (1998a,b,c) determined apparent $K_{\mathrm{m}}$ values for several azo, phthalocyanine and anthraquinone dyes including RBBR; those values are close to the values obtained in our experiments.

The RBBR decolourisation takes place in the absence of exogenous Mn(II). Furthermore, after several purification steps, it was eventually possible to isolate the main enzyme responsible for that decolourisation process. However, the lignin peroxidase isolated from this strain was not able to oxidise RBBR directly. The enzyme that accounts for this capacity is able to oxidise manganese, as well as veratryl alcohol and 2,6-dimethoxyphenol in a manganese-independent reaction, and has a high affinity to RBBR; it corresponds to the newly described third peroxidase, which was already mentioned above. This fact sugests that this enzyme is the one responsible for the high efficiency of decolourisation by this strain that is observed in vivo.

\section{Acknowledgements}

P.R.M. was sponsored by a doctoral fellowship granted by FCT (PRAXIS XXI/BD/15825/98, Portugal).

\section{References}

Azmi, W., Sani, R.K., Banerjee, U.C., 1998. Biodegradation of triphenylmethane dyes. Enzyme Microbiol. Technol. 22, 185-191.

Camarero, S., Sarkar, S., Ruiz-Duenas, F.J., Martínez, M.J., Martínez, Á.T., 1999. Description of a versatile peroxidase involved in the natural degradation of lignin that has both manganese peroxidase and lignin peroxidase substrate interaction sites. J. Biol. Chem. 274, 10324-10330.

Castillo, M.P., Stenström, J., Ander, P., 1994. Determination of manganese peroxidase activity with 3-methyl-2-benzothiazolinone hydrazone and 3-(dimethylamino) benzoic acid. Anal. Biochem. 218, 399-404.

Heinfling, A., Martínez, M.J., Martínez, A.T., Bergbauer, M., Szewzyk, U., 1998a. Transformation of industrial dyes by manganese peroxidases from Bjerkandera adusta and Pleurotus eryngii in a manganese-independent reaction. Appl. Environ. Microbiol. 64, 2788-2793.
Heinfling, A., Ruiz-Duenas, F.J., Martínez, M.J., Bergbauer, M., Szewzyk, U., Martínez, A.T., 1998b. A study on reducing substrates of manganese-oxidizing peroxidases from Pleurotus eryngii and Bjerkandera adusta. FEBS Lett. 428, 141-146.

Heinfling, A., Martínez, M.J., Martínez, A.T., Bergbauer, M., Szewzyk, U., 1998c. Purification and characterization of peroxidases from the dye-decolorizing fungus Bjerkandera adusta. FEMS Microbiol. Lett. 165, 43-50.

Kaal, E.E.J., Jong, E., Field, J.A., 1993. Stimulation of ligninolytic peroxidase activity by nitrogen nutrients in the white rot fungus Bjerkandera spp. strain BOS55. Appl. Environ. Microbiol. 59, 4031-4036.

Kim, B., Ryu, S., Shin, K., 1996. Effect of culture parameters on the decolorization of remazol brilliant blue $\mathrm{R}$ by Pleurotus ostreatus. J. Microbiol. 34, 101-104.

Knapp, J.S., Newby, P.S., Reece, L.P., 1995. Decolorization of dyes by wood-rotting basidiomycete fungi. Enzyme Microbiol. Technol. 17, 664-668.

Kotterman, M.J.J., Wasseveld, R.A., Field, J.A., 1996. Hydrogen peroxide production as a limiting factor in xenobiotic compound oxidation by nitrogen-sufficient cultures of Bjerkandera spp. strain BOS55 overproducing peroxidases. Appl. Environ. Microbiol. 62, 880-885.

Linko, S., Haapala, R., 1993. A critical study of lignin peroxidase activity assay by veratryl alcohol oxidation. Biotechnol. Tech. 7, 75-80.

Mester, T., Field, J.A., 1997. Optimization of manganese peroxidase production by the white rot fungus Bjerkandera spp. strain BOS55. FEMS Microbiol. Lett. 155, 161-168.

Mester, T., Field, J.A., 1998. Characterization of a novel manganese peroxidase-lignin peroxidase hybrid isozyme produced by Bjerkandera spp. strain BOS55 in the absence of manganese. J. Biol. Chem. 273, 15412-15417.

Mester, T., Pena, M., Field, J.A., 1996. Nutrient regulation of extracellular peroxidases in the white rot fungus Bjerkandera spp. strain BOS55. Appl. Microbiol. Biotechnol. 44, $778-784$.

Ollikka, P., Alhonmäki, K., Leppänen, V., Glumoff, T., Raijola, T., Suominen, I., 1993. Decolorization of azo, triphenyl methane, heterocyclic and polymeric dyes by lignin peroxidase isoenzymes from Phanerochaete chrysosporium. Appl. Environ. Microbiol. 59, 4010-4016.

Ollikka, P., Harjunpää, T., Palmu, K., Mäntsälä, P., Suominen, I., 1998. Oxidation of crocein orange $\mathrm{G}$ by lignin peroxidase isoenzymes. Kinetics and effect of $\mathrm{H}_{2} \mathrm{O}_{2}$. Appl. Biochem. Biotechnol. 75, 307-321.

Palma, C., Martínez, A.T., Lema, J.M., Martínez, M.J., 2000. Different fungal manganese-oxidizing peroxidases: a comparison between Bjerkandera spp. and Phanerochaete chrysosporium. J. Biotechnol. 77, 235-245.

Pasti, M.B., Crawford, D.L., 1991. Relationship between the abilities of streptomycetes to decolorize three anthron-type dyes and to degrade ligninocellulose. Can. J. Microbiol. 37, 902-907.

Ruiz-Duenas, F.J., Martínez, M.J., Martínez, Á.T., 1999. Molecular characterization of a novel peroxidase isolated 
from the ligninolytic fungus Pleurotus eryngii. Mol. Microbiol. 31, 223-235.

Swamy, J., Ramsay, J.A., 1999. The evaluation of white rot fungi in the decoloration of textile dyes. Enzyme Microbiol. Technol. 24, 130-137.

Shin, K., Oh, I., Kim, C., 1997. Production and purification of remazol brilliant blue $\mathrm{R}$ decolorizing peroxidase from the culture filtrate of Pleurotus ostreatus. Appl. Environ. Micro- biol. 63, 1744-1748.

Spadaro, J.T., Renganathan, V., 1994. Peroxidase-catalyzed oxidation of azo dyes: mechanism of disperse yellow 3 degradation. Arch. Biochem. Biophys. 312, 301-307.

Vyas, B.R.M., Molitoris, H.P., 1995. Involvement of an extracellular $\mathrm{H}_{2} \mathrm{O}_{2}$-dependent ligninolytic activity of the white rot fungus Pleurotus ostreatus in the decolorization of remazol brilliant blue R. Appl. Environ. Microbiol. 61, 3919-3927. 